\title{
Tracking Technology Lessons Learned in Two Health Care Sites
}

\author{
Jeff Craighead, $\mathrm{PhD}^{1}$, Mary Elizabeth Bowen, $\mathrm{PhD}^{1}$, Angel Klanchar, $\mathrm{RN}^{1}$, \\ Jan Jasiewicz, $\mathrm{PhD}^{1}$, Chadwick A. Wingrave, $\mathrm{PhD}^{2}$ \\ ${ }^{1}$ James A. Haley Veterans Hospital, Tampa, FL; ${ }^{2}$ University of Central Florida, Orlando, FL
}

\begin{abstract}
The ability to track and monitor individual staff and patients presents new opportunities for improving workflow, patient health and reducing health care costs. A real-time locating system (RTLS) is introduced in both a long-term care and a polytrauma transitional rehabilitation program (PTRP) in a Veterans Hospital to track patient and staff locations. Five lessons learned are presented from our experiences and responses to emergent technological, workrelated and social barriers to adoption. We conclude that successful tracking in a health care environment requires time and careful consideration of existing work, policies and stakeholder needs which directly impact the efficacy of the technology.
\end{abstract}

\section{Introduction and Background}

By 2020, the number of Americans age 65 and older will increase from 40 to 55 million $^{1}$. The associated health care costs (Medicare) of this growing elderly population are projected to increase from $\$ 556$ billion in 2011 to $\$ 922$ billion in $2020^{2}$. Emerging models of health care ${ }^{3}$ suggest that the Information Technologies (IT) infrastructure of health systems will need to adapt to these changes and the shortage of nursing staff ${ }^{4,5}$. A real-time locating system (RTLS) that can track and monitor multiple patients at once may begin to address some of these emergent needs in health care by increasing patient monitoring capabilities, reducing the staff/patient ratio and controlling rising health care costs.

While RTLSs have largely been used to track asset movement, such as goods in a warehouse ${ }^{6}$, health care applications may include objective measures to detect falls $\mathrm{s}^{7}$ among institutionalized older adults and prompting rehabilitation patients to perform basic Activities of Daily Living (ADL; ability to keep medical appointments, therapy, medication, bathing, and eating). To determine whether such a system is feasible, useful, and cost effective in practice, other issues such as staff and patient adoption and compliance to the technology must first be addressed.

This report focuses not on the research aspects, but our lessons learned with respect to adoption and compliance of such a RTLS to track the movement of Registered Nurses (RNs), other health care staff, and patients in both a longterm care unit and polytrauma transitional rehabilitation program (PTRP) in a Veterans Hospital. The long-term care patient population consists of adults age 65 and older that are at risk for falls. The PTRP patient population consists of younger (mean age 30 years) adults with memory impairments consistent with traumatic brain injury (TBI) and some physical limitations. In long-term care, the RTLS system was used to track the real-time location and movement of 5 staff (for 30 days) and 17 patients (from 30 days to 6 months). In the PTRP, this system was used with 10 healthcare staff and 10 patients for the purpose of tracking patient compliance performing everyday ADL. The (Ubisense) tracking system used in these facilities is an ultra wide-band RTLS, shown in Figure 1. A RTLS is similar to passive radio frequency identification (RFID) in most aspects, however the tag itself is powered giving it a wider range and the spatial resolution of the system is on the order of 6 inches, versus 36 inches or more for a typical passive RFID system. A previous laboratory study showed that this resolution is sufficient for detecting falls ${ }^{7}$ and previous studies at an assisted living facility showed that the system could be used to track patients with a high degree of accuracy ${ }^{8,9,10}$. However, these studies only looked at the technological feasibility of tracking not the critical adoption issues that must be overcome for deployment in health care settings ${ }^{11,12,13}$.

We believe that this report details a valuable first step - notably, if key players reject the technology and its application the system will not be used. In the short-term, this study's findings inform new and progressive staff and patient-focused technologies designed to support the independence of institutionalized older adults as well as memory-impaired populations. In the long-term, the findings may be used to reduce the health care costs associated with population aging and the need for increased patient monitoring and surveillance by health care workers.

\section{Long-Term Care Facility}

In the long term care facility, RNs were recruited to test the feasibility of the system and to ensure that the RTLS accurately tracked location and movement in a health care setting, as it did in the lab ${ }^{7}$. Enrolling RNs also served to 
make some health care staff aware of the research being conducted on the unit and it was hoped that this would increase patient compliance in the next stage of the research. Contact with each RN was made individually by a researcher, accompanied by the head nurse, who explained the technology, the study, answered their questions and asked for their consent to be tracked. The hang tag was attached to the RN's nametag, required to be worn while on duty, so RNs did not have to remember to wear the tag every day. This worked out well - with $100 \%$ compliance likely because the hang tags fit into their existing routine. Five RNs were tracked throughout a hallway, nursing station and shower facility in real-time, continuously over the course of 30 days with ceiling-mounted sensors (see Figure 1). The RTLS recorded the (x,y, and $\mathrm{z}$ ) coordinates of tags with respect to a fixed location in the facility with an average accuracy of 50 centimeters. After 30 days of testing with RNs, 17 fall risk patients were recruited on the unit to wear wrist tags. Patients and staff preferred that patients wear a compact tag on the wrist (see Figure 1) as these were more convenient. Over the course of six months, patient wrist tags were constantly monitored and patient falls were examined using an algorithm previously developed by the researchers?

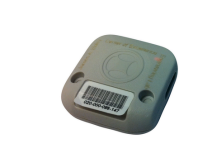

Compact Tag

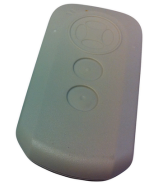

Hang Tag

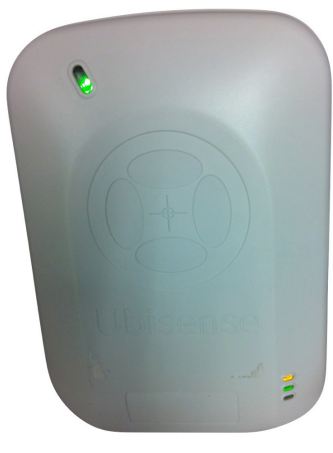

Sensor

Figure 1. Ubisense sensor (about 8" tall) and two tags used in the study (a wrist "compact tag" and "hang tag" worn around the neck). The tags emit an ultra-wide band radio signal that is triangulated by multiple sensors in the environment. The tags are worn by participants to track their location and movement in real-time.

Lessons Learned

We have extracted four lessons learned from our experiences in the long-term care facility.

\section{- Expect equipment installation to require additional time and effort due to policies, safety and layout.}

Any new hospital equipment or device must pass inspection with the appropriate stakeholders - including the electrical, construction and infection departments. Also, because RTLS emits signals into the environment that may interfere with existing medical equipment, it is necessary to monitor the unit's equipment after the system is up and running. It is also useful to discuss this potential interference with staff so that malfunctioning equipment is reported. Space in a healthcare setting is also at a premium, so expect difficulties obtaining it. For us, we required a small space at a nursing station for the RTLS's central hub and data collection laptop, and even this was difficult to acquire on a semi-permanent basis. Lastly, hospital layouts are designed for patients and their health care needs, and may not be ideal for RTLS technology. Multiple sensors triangulate tag positions but the long hallways, thick walls and crowded above-the-ceiling space required additional sensors in this study and made stringing cable difficult.

- Expect stakeholders in the environment to develop concerns and even myths about the technology.

Others do not always understand the technology like you. The sensors, shown in Figure 1, may be mistaken for a camera when installed in a corner or a hallway. It was not uncommon to be asked about this "surveillance" device and whether its purpose is to videotape unit activity. Other technology myths that had to be addressed over the course of the study included whether the tag was a listening device or was able to restrict the movement of the wearer. For example, one patient declined continued participation in the study after two months because they believed the device closed elevator doors prematurely. Some of these problems may be due to our older adult patient population who may be less familiar with new technology ${ }^{14,15}$. However, older adults were not the only ones to express concerns. The most common concern of the recruited RNs was that the technology was designed to track their work habits - recording their work hours and the location, frequency and time spent on breaks. There was little communication about the technology among the staff so this myth persisted across the $30 \mathrm{RN}$ tracked days. 
- Expect to spend time in the stakeholder's environment to inform them, increase compliance and acceptance.

The research will be important to you - but not necessarily to others. Though VA leadership and management approved the installation of the RTLS and five RNs agreed to participate in the study, the project and its goals were not clearly communicated to all of the nursing staff and patients on the unit.

To inform the nurses, three in-service training sessions (morning, evening, and night shift) were conducted during the patient study to ensure that all nursing staff understood the research. However, these in-services did not gain the trust or compliance that we expected - likely because nursing staff began to view the project as a "research feasibility study" that had no direct impact on patient care or their workflow. As a result, we found that enrolled patients were less likely than before to be wearing their wrist tags; nursing staff were no longer replacing the wrist tags on the patients when they had been taken off (e.g., for bathing).

To increase compliance, an RN researcher began to visit the unit three times a week to check patient wrist tags. The RN researcher appealed to the nurses' and patients' needs. When patients were found untagged, the root cause was assessed and the patient was retagged. If the patient stated that their nurse had not replaced the tag, the RN researcher contacted the nurse to reinforce their knowledge of the project and address any issues or concerns.

To increase acceptance, the RN researcher appealed to the nurses' and patients' needs. The project's potential to improve health care was discussed with nurses in their own vernacular. Patients were happy to interact with someone regularly, which increased their motivation to be part of the study and appealed to their sense of duty to fellow veterans. This created project advocates that talked to their cohorts about the study and led to unsolicited patients offering to join the study. Thus, the RN researcher, due to her clinical knowledge and experience in a health care environment, was better able than the other researchers to gain rapport with staff and patients.

- Expect to iterate on your research protocol to better incorporate your technology with stakeholder needs.

Local and site-specific workflows, policies and interpretations, that you may be unaware of, will impact the research. Just as the above in-service informative sessions with nurses led to an unexpected drop in compliance, make a best guess on how to incorporate your technology, be watchful, respectful and reactive, looking for any change you make to impact other areas of your research. We have two additional examples of this at this site:

Example 1: At first, Velcro bands held the tags on patient wrists but this material quickly became dirty. We then tried colored medical bands, typically used to alert staff to a chronic condition or an adverse event risk. However, pink was the only color not in use and this color was an issue for some of the older males in the study. We switched to yellow, which was already in use as a falls risk indicator. This worked well as the yellow bands afforded the tag's purpose and were better received by the patients.

Example 2: Our initial policy was that the tag was to be removed during patient showers and baths. However, we found that tags were lost or removed when removed for showers or baths and nurses did not replace them on the patient. Additionally, patients who left the facility with family or friends or were admitted to the adjoining hospital for care rarely came back with their tag. The policy was changed to remove bands when the patient left facility grounds. Upon return the band was waiting to be put back on as part of the patient's check-in procedure. Additionally, the tag removal policy for baths was dropped, saving the nurses the repeated hassle. To improve the tag's water resistance, the tags' cases were opened and re-sealed with petroleum jelly.

\section{Discussion}

Over the course of the unit's activities, we extracted four lessons learned that we expect to be applicable to other tracking studies in a health care environment. These lessons regard equipment installation, stakeholder concerns and myths, researcher time and protocol/policy iteration. Because of these lessons, we were eventually able to successfully track patients and RNs in an ALF. Additionally, the nursing staff suggested that health care professionals be provided with a real-time mapping system that detailed the location and movement of their patients. Such a map could help staff ensure that the system is working and that the patients are located in desired areas. If each individual patient were identified in the map it would also provide nursing staff with information on at risk patients, such as those at risk for falls. Following their suggestion, the next study implemented the map..

\section{Polytrauma Transitional Rehabilitation Program Facility}

The Polytrauma Transitional Rehabilitation Program (PTRP) is a relatively new facility, with the goal of providing comprehensive rehabilitative therapy for up to 10 Traumatic Brain Injury (TBI) 10 patients in a communal-type living environment. In this 5 apartment, 10 bed, 41 room facility (including staff offices and communal areas) we 
used163 sensors to track patients and staff with multiple sensors located in patient rooms, hallways and common areas, providing coverage of the entire facility. The RTLS system was installed as part of a location aware "smart home" designed to provide PTRP patients with a cognitive prosthetic to augment the rehabilitative care provided by PTRP staff ${ }^{10}$. The average stay for PTRP patients is approximately 4 months with the mean age being 30 years old -a considerably different demographic than the long-term care facility. Over the last 6 months, we tracked 10 patients (from 30 days to 4 months) as well as 10 staff members (up to six-months).

The target trauma of this facility's population is a TBI that is partly recoverable through the comprehensive rehabilitation program provided at the facility. The benefits of location tracking for this population are the ability to prompt and guide them when they have difficulties performing ADL as well as maintain their scheduled medical appointments. In addition, we are installing touch screen computers at regular intervals and introducing other mobile devices to interact with the location data as part of a longer-term effort. This is consistent with our goal of augmenting the rehabilitation in the unit through tracking, behavior compliance and progress monitoring.

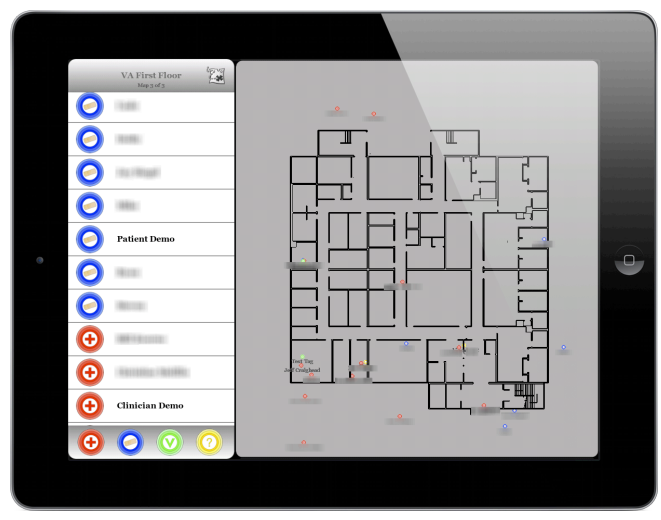

Figure 2. The iPad PTRP Map application displays patient and staff location on a facility map. This application was incorporated into workflows and increased visibility and understanding of the RTLS technology in the PTRP.

\section{Applying our "Lessons Learned" in the PTRP}

Due to the increased number of sensors required for this project and the amount of time installation and calibration took in the long-term care unit, Ubisense and our VA Facilities Department installed the hardware for this system instead of the research team. Installation took about 3 months. The researchers used this start-up time to lead weekly focus groups with all of the health care staff in the PTRP, informing them of the benefits of this technology. We also worked with staff to design applications to help patients with their ADL and to improve staff workflow. As expected, the staff in the PTRP expressed initial concerns about the technology - whether cameras were being used and whether patients would accept it - and they were also concerned about the sensor aesthetics. Patients did have concerns about the technology but meetings between the patients, staff and researchers explained the technology. There was one refusal, an older adult, but most patients were accepting and interested, possibly due to the (overall) younger patient population in PTRP. Staff and patient compliance has not been a problem thus far. This may be because the focus groups addressed concerns early and often - putting stakeholders at ease and allowing us to iteratively improve our proposed rehabilitation applications at their site. For instance, we worked with Ubisense to improve the aesthetics of the sensors for the PTRP and designed a prototype "watch" tag for patients and staff that is more site-appropriate (i.e. medical bands can appear less dignified). The watch functions as a timepiece and tracking device, aligning with the PTRP goals of increasing medical appointment adherence and independence.

\section{Map Application}

Based on the feedback of the long-term care unit and PTRP staff, our first prototype application was an iPad-based map of the PTRP facility (see Figure 2) that enabled the staff to quickly locate patients in the facility. This simple application allowed us to test the installation and demonstrated the technology's operation. Staff reports were positive, finding it a useful time-saver. The map application has been in use for about four months and receives regular updates to improve performance and implement staff feature requests.

- $\quad$ Expect glitches and delays to impact a system's perception and uptake.

An additional lesson learned is regarding interest and research timelines. Due to a combination of problems with custom hardware and software bugs, some of the initial participant enthusiasm was lost. Future applications will be 
tested in a separate location to ensure that the "kinks" have been worked out and the initial rollout will include a beta testing period with participant education.

\section{Conclusions and Future Work}

Five lessons learned have been created from our experiences in incorporating tracking technology in two health care environments with differing patient populations, needs and goals. For those working in similar health care settings, researchers should expect: (1) equipment installation to require additional time and effort due to policies, safety and layout; (2) stakeholders in the environment to develop concerns and even myths about the technology; (3) to spend time in the stakeholder's environment to inform them, increase compliance and acceptance; (4) to iterate on your research protocol to better incorporate your technology with stakeholder needs; and (5) glitches and delays to impact a system's perception and uptake. Tracking technologies enable new opportunities in health care settings, allowing nearly all participants can gain in some way so long as the researchers are vigilant and develop robust applications. Future work includes motivating beneficial behaviors in recovery, recognizing declining (or improving) health patterns, alerting nursing staff of safety concerns and evaluating treatment or training efficacy.

\section{Acknowledgements}

The authors' views are their own and do not necessarily represent the views of the VHA. This research was supported by a grant from the National Center for Patient Safety.

\section{References}

1. U.S. Department of Health and Human Services. A Profile of Older Americans: 2010. Washington, D.C. 2010.

2. Sisko AM, Truffer CJ, Keehan SP, Poisal JA, Clemens MK, Madison AJ. National health spending projections: the estimated impact of reform through 2019. Health Aff (Millwood). Oct 2010;29(10):1933-1941.

3. Varshney, U. Pervasive Healthcare. 2003. Computer. Vol. 36. No. 12, pp. 138-140.

4. Buerhaus PI, Auerbach DI, Staiger DO. The recent surge in nurse employment: causes and implications. Health Aff (Millwood). Jul-Aug 2009;28(4):w657-668.

5. Institute of Medicine. Retooling for an aging America: Building the health care workforce. 2008.

6. Ubisense. Real-Time Location Fact Sheet. 2009; http://www.ubisense.net/pdf/factsheets/products/software/Real-time-Location-EN090908.pdf.

7. Bowen M, Craighead J, Wingrave C, Kearns W. Real-time locating systems (RTLS) to improve fall detection. Gerontechnology. 2010;9(4):445-452.

8. Kearns W, Nams V, Fozard J. Wireless fractal estimation of tortuosity in movement paths related to cognitive impairment in assisted living facility residents. Methods in Information Medicine 2010;49(6):592-598.

9. Kearns W, Fozard J, Nams V, Craighead J. "Wireless telesurveillance system for detecting dementia". Journal of Gerontechnology. In Press. 2011.

10. Jasiewicz J, Kearns W, Craighead J, Fozard J, Scott S, McCarthy J Jr. Smart Rehabilitation for the $21^{\text {st }}$ Century. Journal of Rehabilitation Research and Development. In Press. 2011.

11. Bardram J, Hansen T, Mogensen M and Soegaard, M. Experiences from real-world deployment of contextaware technologies in a hospital environment. 2006. UbiComp 2006: Ubiquitous Computing. pp. $369--386$.

12. Hansen, Thomas R., Bardram, Jakob E., and Soegaard, Mads. Moving Out of the Lab: Deploying Pervasive Technologies in a Hospital. 2006. IEEE Pervasive Computing. Vol. 5. Issue 3. pp. 24-31.

13. Poon E, Blumenthal D, Jaggi T, Honour M, Bates D and Kaushal R. Overcoming Barriers To Adopting And Implementing Computerized Physician Order Entry Systems In US Hosp. Health Affairs, 23, 4 (2004):184-190.

14. Morrell RW, Mayhorn CB, Bennett J. A survey of World Wide Web use in middle-aged and older adults. Hum Factors. Summer 2000;42(2):175-182.

15. Zimmer Z, Chappell N. Receptivity to new technology among older adults. Dis. Rehab. 1999;21(5-6):222-230. 